Caries Res 1995;29:523-524

\title{
Subject Index Vol. 29, 1995
}

Acid production 402

Acid-base balance 20

Acidogenic potential index 75

Acidogenicity 68

Acidosis 20

Acidulated phosphate fluoride 331

- - gel 327 Actinomyces 507

naeslundii 159 Adhesion 470

Age, fluoride distribution 218

Air-drying 14,231

Aluminum 237,490

Amelogenesis 20

Amine fluoride solution, topical application

frequency 168 Ammonium chloride 20 Anticaries clinical trial 163 Antimicrobial varnish 188

Approximal caries 364

- $\quad$-, aetiology 42

Artificial enamel lesions, structure 122

Backscattered electron imaging 111 Bacterial composition 46 Bicarbonate dentifrices 143 Bitewing radiography 81 Blackcurrant drink 75 Bone 20 Brushite 258

Caffeine 285

Calcium fluoride 122,223

phosphates 223 Caries 327, 470, 477

diagnosis 2, 8, 88

incidence 148

prediction 148, 343

progression 449

risk 272,455

Cariogenic potential, chocolate 418

Cariogenicity 68

Cariostatic agents 62

Casein 498

Cell death 181

Chemomechanical caries removal 111

Chewing gum(s) 477

- $\quad$-, sugar-free, urea content 172

Child(ren) 163,280,343,449

Chlorhexidine 62,159,413

Chlorhexidine-fluoride mouthrinses 181

Citrate 75 
Citric acid 467

Clearance 467 Cohort study 272 Collagen 192,483 Collagenase 483 Colorimeter 251 Confocal microscope 198 Contact microradiography 107 Cytoplasmic membranes 413

Decalcification 285 Demineralization 204, 285, 337 Dental caries 168, 343, 359, 431 , 435, 449, 455,461

- prevention 331

education 355

enamel 210,223,258

fluorosis 251

plaque $54,172,181,188,382$

- microflora 154

prophylaxis 331

radiology 355 Dentifrice 435

Dentine 8, 14, 107, 118, 154, 192,231,337

caries 111

proteins 483 Diagnosis 243, 364, 377 -, laboratory 359

-, secondary caries 371 Dietary habits 343 Dissolution 130

- $\quad$ rate 513

Drinking water, fluoride levels 218 Drying 118

Ecology 159

Electrical conductance 88,94,100

resistance monitor 81 Electron probe microanalysis 111 Electronic control 418 Enamel

$8,20,88,285,337,513$

defects 137

demineralization 68

dissolution 490

erosion 467

maturation 94

morphology 251 Enzymes 498 Epidemiology 449,461 Erosion 349,470

Fibre-optic transillumination 364 Fissure sealant 377 Fluorhydroxyapatite 130

Fluoride(s) 20, 130, 163,210,231,237, 258,431,435,442,477

concentration 168

dentifrice 337

distribution, rat molar cementum, age 218

excretion 26, 35

ingestion 137

monitoring 26, 35

release 424 -, topical 223

-, application 331

uptake 424

varnish 327 Fluorosis 20, 137 Fruits 68

Glass ionomer 50

- $\quad$ - cements 424

Glucan(s) 498, 507

Glucosyltransferase 498 
Glycolysis 181

Glycoproteins 483

Grey discolouration 371

Hidden caries 46

Histology 243

Hydroxyapatite 122,210,455,507

Immunoblotting 483

Immunofluorescence 198

Infants 343

Infection 461

Initial enamel caries lesions, changes 2

Interference 159

Lactobacilli 50, 143 Lactobacillus 42 Laser 192

fluorescence 2

irradiation 513 Leakage 100 Lesion shrinkage 14 Light absorption 8

scattering 8

Macroscopy 243 Magnesium 20 Marginal fracture 371 Melibiose 407 Metered feeding 418

Microbiology 272, 382 Microhardness 349

Microradiography 204, 359

Microscopy 359

Milk 498

Mineral content 111

Mineralization 285

N-Monochloro-DL-2-aminobutyricacid

111 Mouthrinse 431 Mouthrinsing 266 Mutans streptococci 46, 50, 62, 143, 188,

$198,280,343,396$

NaF dentifrice 163

Occlusal caries 243, 377

- detection 355

-, diagnosis 94

fissure caries, in vivo diagnosis 81 Oral hygiene 266

sucrose clearance 396 Orthodontic appliances 188

pH recovery, urea 172 Phosphoproteins 483 Plaque 75

composition 442

fluoride 50

$\mathrm{pH} 75$

Polarized light microscopy 204 Polymerase chain reaction 407

Preschool children 148, 272, 280, 435 Prevention 431 Primary dentition 272 Protein adsorption 210 Proteoglycans 483

Radiographic caries diagnosis 355

Radiography 243, 359, 364

Rat caries, aluminum inhibition 237

- $\quad$ saliva 507

Recurrent caries 266

Remineralization 204,231,337,477

Ribotyping 407 
Root caries 14,154,382

- $\quad$ - models 204

Saliva 54, 62, 280, 467, 477

- $\quad$ fluoride 50

Salivary mutans streptococci 148

Salt fluoridation 35

Scanning electron microscopy 251,513

Sealant 100

Secondary caries 100,198,266

Shrinkage 118

prevention 107 Single section 231 Starches 68 Streptococcus 507

gordonii 159

mitis 159

mutans 42, 54, 159, 198, 402, 407, 413, 442, 455

-, lethal photosensitization 192

oralis 159

sanguis 159

sobrinus 42, 54, 402 Sucrose 477

retention 396 Sugar restriction 54 Surface characteristics 210

microhardness 513 Surfactants 490

Teeth, primary 449

Tooth demineralization 223

Toothbrushing 266,280, 337,435

Toothpaste 442

Triclosan 163

Trypsin 483

Tubules 118

Urinary fluoride 26,35

Validity 364

Visual inspection 364

Water fluoridation 137

- $\quad$ rinsing 337, 435

524

Subject Index Vol. 29, 1995 